\title{
LAS CONDUCTAS DISRUPTIVAS EN EL SISTEMA ESCOLAR ESPAÑOL. EVOLUCIÓN LEGISLATIVA
}

\author{
Disruptive behaviour in the spanish school system. Legislative development \\ Inmaculada Saco Lorenzo, Ignacio González López, \\ María Amor Martín Fernández y Purificación Bejarano Prats.
}

Fecha de recepción: 01/12/2019

Fecha de aceptación: 15/12/2019

RESUMEN: En la actualidad el incremento de los problemas de disciplina en el aula supone una preocupación para el profesorado y un obstáculo para el desarrollo del proceso de enseñanza-aprendizaje. Para que la intervención educativa sea eficaz, es necesario diferenciar los distintos tipos de conflictos, abordándolos desde diversas perspectivas. En este artículo se ha realizado un análisis documental de las sucesivas leyes educativas españolas, buscando en cada una la frecuencia de aparición de palabras claves referidas al comportamiento del alumnado, se ha analizado la respuesta ante la conflictividad y la diferencia entre el abordaje de la conducta grave y la disruptiva, menos grave. Como resultado se observa una evolución positiva de la normativa, influida por una sociedad más concienciada de la importancia de un buen clima escolar para conseguir el sano desarrollo personal y mejor aprendizaje del alumnado. La ley actual diferencia entre conductas graves y leves y muestra una mayor preocupación, no solo por frenar el incremento de estos comportamientos, sino también por prevenirlos. Pero todavía queda mucho por avanzar, pues aún no se dan orientaciones claras que ayuden al profesorado a abordar las conductas leves de forma adecuada, pese a las dificultades que suponen para el proceso de aprendizaje.

PALABRAS CLAVE: legislación educativa; disciplina; conflicto; sistema escolar; conducta disruptiva

ABSTRACT: Nowadays, increasing discipline problems in the classroom have become a concern for teachers and an obstacle for the development of the teaching-learning process. To adequately develop an effective educational intervention, it is essential to distinguish between different types of conflicts and to approach them from different perspectives. This paper presents an analysis of the successive educational Spanish laws, specifically examining the frequency of appearance of those keywords regarding students' behavior in each law. The responses provided to approach conflict were studied, differentiating from serious misconduct and less serious disruptive behavior.Results show a positive evolution of the Spanish regulations: This is due to the increase of social awareness regarding the importance of creating a favourable school environment to ensure a healthy personal development and better learning. The current law distinguishes misconduct and disruptive behavior and also expresses its concern about the increase in this kind of behavior and provides ways of preventing it. However, further progress is still to be achieved as there is a need for clear guidelines for teachers to properly deal with disruptive behavior. Despite the difficulties that this kind of behavior entail to the learning process, these recommendations are still not laid down despite the difficulties faced by the learning process. \section{behaviour}

KEY WORDS: educational legislation; discipline; conflict; school system; disruptive 


\section{Introducción}

En los últimos años, la sociedad ha sufrido vertiginosos cambios que han transformado la manera de pensar y vivir de la población en general, afectando al entorno de la comunidad educativa y reflejándose en el comportamiento de una parte del alumnado, que manifiesta mayor inseguridad, menor resistencia al fracaso y falta de capacidad de esfuerzo (De la Fuente, 2012). La variabilidad de personas que conviven en el aula y el largo tiempo que estas permanecen juntas son otros factores que propician la aparición de conflictividad (De Souza, 2009).

Esto ha provocado una creciente preocupación en el profesorado, que dedica más de un 13\% del tiempo en el aula a la resolución de problemas de disciplina, como recoge el informe TALIS, realizado por la Organización para la Cooperación y el Desarrollo (OCDE, 2014). Además, los resultados de recientes investigaciones han confirmado que la conflictividad perjudica de forma inevitable al aprendizaje del alumnado (Aguirre, Fernández y Silvero, 2016; Gotzens, Cladellas, Clariana y Badía, 2015; Valderrama, 2017). Por ello, el problema de la indisciplina en el aula se debe afrontar como una oportunidad para mejorar el funcionamiento cotidiano de la organización escolar (Villalba, 2016; Gómez y Cuña, 2017). Para que la intervención educativa sea eficaz, es necesario que se diferencien los distintos tipos de comportamientos negativos, pues se observa que, en muchas ocasiones, se generaliza al hablar de conducta disruptiva, haciendo referencia a comportamientos muy distintos, difíciles de abordar desde una misma perspectiva (Esteban, Fernández y Díaz, 2012). En este sentido, como afirman Gotzens, Badía, Genovard y Dezcallar (2010), se debe diferenciar entre las conductas de indisciplina de tipo convencional -aquellas que tienen que ver con el incumplimiento de normas y reglas establecidas por la sociedad, cuyas consecuencias son más graves, como maltrato, bullying o agresiones- y las de tipo instruccional que serían las llamadas disruptivas, comportamientos como hablar sin permiso, levantarse, interrumpir, gritar o tirar bolas de papel, que impiden el buen funcionamiento del aula (Gotzens et al., 2015; Badía, Cladellas, Gotzens y Muntada, 2012).

El abordaje de las conductas disruptivas es decisivo para conseguir un adecuado clima de aula y un satisfactorio proceso de enseñanza-aprendizaje, por lo que es preciso identificar la respuesta que ha dado la normativa española a esta problemática a lo largo del tiempo.

Por ello, el objetivo de este artículo es analizar e identificar la evolución y variación de dicha respuesta en la legislación educativa. Para tal propósito, se ha realizado una revisión y análisis de la normativa del territorio MEC, sin mencionar lo establecido por las comunidades autónomas, observando su evolución en cuanto al tratamiento de la conflictividad en la escuela por las variaciones que se han sucedido, debido a cambios sociales, políticos y económicos de cada época. Además, se han identificado las funciones que los distintos miembros de la comunidad educativa han tenido en cada una de las leyes examinadas en la resolución de conflictos.

Esta revisión constata que no aparece el término "conducta disruptiva" como tal, entendido como el comportamiento del alumnado que interrumpe e impide el normal desarrollo de la clase. Por ello, se ha decidido hacer un análisis documental de aquellos términos con significado similar o que puedan confundirse con el concepto estudiado. Así se han examinado: conducta y comportamiento (utilizados como sinónimos), disciplina, conflicto, violencia, conducta disruptiva y falta leve y grave, identificando las leyes en las que aparecen, número de veces y significado de cada uno de ellos. No se hace alusión a otros conceptos como convivencia o rendimiento académico ya que estos no forman parte del 
objeto de este estudio.

\section{Los problemas de conducta en la legislación educativa española}

A principios del siglo XIX se produce en España un gran avance en el plano educativo, cuando en 1812 las Cortes de Cádiz aprueban la primera Constitución, que propugna que la Educación Primaria debe ofrecerse a toda la población, unificando los planes de enseñanza para todo el Estado (MECD, 2004). Se conseguirá la estabilidad en materia de educación en septiembre de 1857, con la aprobación de la Ley de Instrucción Pública, primera ordenación general de todas las enseñanzas del sistema educativo español (Montero, 2009). En este momento, no parece haber preocupación legislativa por el mal comportamiento en la escuela.

Será en 1970, con la aprobación de la Ley General de Enseñanza (LGE), cuando por primera vez se hace referencia a la falta de disciplina del alumnado, advirtiendo que se puede perder la condición de estudiante de forma temporal o definitiva como consecuencia de esta conducta. Posteriormente, la Ley Orgánica de Estatutos de Centros Escolares (LOECE) de 1980, hace referencia a los órganos de gobierno que deben hacerse cargo de sancionar el mal comportamiento, y añade el tipo de sanción que se debe aplicar para las faltas graves. Sin embargo, no establece ninguna indicación para los problemas de comportamiento leves, entre los que se encuentran las conductas disruptivas. Este aspecto tampoco será abordado por la Ley Orgánica reguladora del Derecho a la Educación (LODE) de 1985, que seguirá insistiendo en las atribuciones del Consejo Escolar en materia de disciplina.

En 1990, se aprueba la Ley Orgánica de Ordenación General del Sistema Educativo (LOGSE), que acoge a toda la población escolar desde los 6 hasta los 16 años. Se incrementó la cifra de alumnado de secundaria, suponiendo una revolución educativa al ofrecer posibilidades a niños y niñas con diferentes capacidades y características personales y sociales. Pero esto ocasionó otros problemas, ya que el profesorado no tenía recursos para atender a un alumnado tan heterogéneo, lo que provocó una gran desmotivación ante la labor docente (De Puelles, 2011). Todos estos factores influirán en el incremento de conductas disruptivas y conflictos, impulsando a la comunidad educativa a tomar conciencia de la importancia de una educación que ayudara a prevenir este tipo de comportamiento, reflejándolo posteriormente en el Real Decreto 732/1995 de 5 de mayo de 1995.

La Ley Orgánica de Calidad Educativa (LOCE), en 2002, insiste en educar a los discentes en el esfuerzo y la responsabilidad como medio para la mejora de la enseñanza, pero antes de que se complete su aplicación, fue derogada y, en 2006, se aprobó la Ley Orgánica de Educación (LOE) en la que prevalece la educación en la prevención y resolución de conflictos para evitar la violencia en todos los ámbitos de la vida personal, familiar y social.

Por último, en 2013 se aprueba la Ley Orgánica de Mejora de la Calidad Educativa (LOMCE), que modifica algunos aspectos de la LOE (2006). En el texto consolidado de ambas leyes se insiste en la atención a la prevención de conflictos y además se otorga a las sanciones un carácter recuperador. Se diferencia entre falta grave y leve, indicando las correcciones ante las graves y cediendo a los centros la función de atender de forma inmediata las faltas leves, entre las que se encuentran las conductas disruptivas, aunque sin dar pautas concretas de corrección.

Para comprobar la evolución en la normativa se han analizado las leyes educativas especificadas en la tabla 1, buscando la aparición de las palabras "conducta, comportamiento, 
disciplina, conflicto, violencia, conducta disruptiva, falta leve y falta grave". Las dos primeras se utilizan como sinónimas para referirse a la reacción que el alumnado manifiesta ante el ambiente escolar; disciplina como la observancia de las leyes educativas; conflicto alude a problemas interpersonales en los centros educativos y violencia a la utilización de la fuerza y la ira contra una persona o cosa. El término conducta disruptiva, definido anteriormente, no se ha encontrado en ninguna de las leyes examinadas. En la LOMCE (2013) se hace referencia a la falta leve, diferenciándola de la falta grave, que la propia ley define como la conducta que "atenta contra la dignidad personal de otros miembros de la comunidad educativa" (LOMCE, 2013, p. 97903). No se han tenido en cuenta otras acepciones de las palabras, como cuando "disciplina" se refiere a materia o área de estudio o "conflicto" a falta de acuerdo entre el titular del centro concertado y el Consejo Escolar del mismo, como aparece en la LODE (1985) y en la LOPEG (1995).

Tabla 1. Leyes educativas españolas y frecuencia de aparición de términos relacionados con las conductas disruptivas

\begin{tabular}{|c|c|c|c|}
\hline Año de aprobación & Ley & Palabras clave & Frecuencia \\
\hline 1857 & Ley de Instrucción Pública & $\begin{array}{l}\text { conducta } \\
\text { disciplina }\end{array}$ & \\
\hline 1970 & LGE & $\begin{array}{c}\text { conducta/comportamiento } \\
\text { disciplina }\end{array}$ & \\
\hline 1980 & LOECE & $\begin{array}{l}\text { disciplina } \\
\text { falta grave }\end{array}$ & \\
\hline 1985 & LODE & $\begin{array}{l}\text { conflicto } \\
\text { disciplina }\end{array}$ & $\begin{array}{l}1 \\
2\end{array}$ \\
\hline 1990 & LOGSE & comportamiento & 1 \\
\hline 1995 & LOPEG & $\begin{array}{l}\text { conflicto } \\
\text { conducta }\end{array}$ & \\
\hline 2002 & LOCE & $\begin{array}{c}\text { conducta/comportamiento } \\
\text { conflicto } \\
\text { disciplina }\end{array}$ & $\begin{array}{l}4 \\
3 \\
9\end{array}$ \\
\hline 2006 & LOE & $\begin{array}{c}\text { conflicto } \\
\text { conducta/comportamiento } \\
\text { disciplina } \\
\text { violencia }\end{array}$ & $\begin{array}{r}16 \\
8 \\
9 \\
9\end{array}$ \\
\hline 2013 & LOE-LOMCE & $\begin{array}{l}\text { conflicto } \\
\text { conducta } \\
\text { disciplina } \\
\text { violencia } \\
\text { falta grave } \\
\text { falta leve }\end{array}$ & $\begin{array}{r}11 \\
5 \\
6 \\
8 \\
1 \\
1\end{array}$ \\
\hline
\end{tabular}

Nota: elaboración propia.

Se advierte en la tabla 1 el incremento de aparición de los conceptos relacionados con los problemas de comportamiento. En la Ley de Instrucción Pública, los términos conducta y disciplina aparecen referidas al buen comportamiento y aprovechamiento del estudio, declarando que la buena conducta y la disciplina académica del alumnado se premiaría, sin embargo, no se contempla la posibilidad de problemas de comportamiento en los centros educativos. En este momento la mayor preocupación es la extensión de la Educación Primaria obligatoria y gratuita. La Ley General de Educación también incide en este tipo de disciplina 
académica, aunque, por primera vez, aparece la alusión al mal comportamiento, instando al profesorado a intervenir y mantener la disciplina si fuera necesario. En esta misma línea se conducen las leyes posteriores con un incremento en la aparición de estos conceptos a partir de la promulgación de la LOCE en 2002, que muestra gran preocupación en un contexto en el que se ha debilitado el valor del deber y el respeto al profesorado, aumentando las agresiones a docentes y entre el alumnado (Pino y García, 2007) y se presenta la disciplina como medio para solucionar esta problemática, sobre todo los comportamientos de carácter grave. Por último, las normativas actuales, LOE (2006) y LOMCE (2013), conscientes de la importancia de sancionar y regular lo relacionado con la violencia de género y los comportamientos de bullying, incrementados en los últimos años (Rodríguez, 2017), se refieren en numerosas ocasiones al tema tratado. Los términos que más veces aparecen son violencia (8) y conflicto (11), refiriéndose a la utilización de estrategias y medidas para conseguir la resolución pacífica de conflictos y una adecuada convivencia en el centro.

\subsection{Los inicios del sistema escolar español y la Ley Moyano de 1857}

La primera normativa estable que regula el sistema educativo español es la Ley de Instrucción Pública, conocida como Ley Moyano, promulgada el 9 de septiembre de 1857 (García, 2017 y Montero, 2009). Ha estado presente más de 100 años, organizando la enseñanza en tres niveles (primaria, secundaria y superior). Pretendió que descendiera la tasa de analfabetismo y para ello estableció la obligatoriedad de la educación de los 6 a los 9 años, aprobando su gratuidad cuando hubiera imposibilidad de pago (Montero, 2009). Pero a pesar de su empeño en proveer de escuelas públicas al país, (art. 105), el nivel de analfabetismo siguió siendo muy alto hasta la primera mitad del siglo XX.

"El objetivo principal de la escuela primaria era lograr que los ciudadanos se condujeran de manera adecuada en la vida" (Domínguez, 2008, p. 270). Por ello, se premiaría la buena conducta del alumnado y la junta de profesores tendría carácter de consejo de disciplina para conocer las faltas académicas de los estudiantes. Se advierte una preocupación por el buen aprovechamiento de la enseñanza, pero no se contempla cómo abordar la conducta disruptiva del alumnado.

\subsection{Ley General de Educación de 1970 (LGE)}

A partir de 1960, en España se produjo un gran desarrollo económico provocado por el traslado masivo de población a las ciudades y la emigración a otros lugares de Europa, surgiendo una clase media más amplia que demandaba la escolaridad. Esta nueva situación conllevó una reforma educativa (Egido, 2002). El encargado de dicha reforma fue Villar Palasí, ministro de Educación y Ciencia, que publicó el Libro Blanco de la Educación con el título "La educación en España, bases para una política educativa" (MEC, 1969), en el que insiste en la educación de la libertad responsable, el fomento de la integración social y la convivencia nacional (Domínguez, 2008). En base a esto, se aprueba la Ley General de Enseñanza (LGE) en 1970, que afirma en su preámbulo que debe tener:

Menor dosis de jurisdicidad (...) y por eso los márgenes y elasticidades que en ella se contienen no deben verse como deficiencias de lo que debe ser una norma sino, por el contrario, como requisitos positivos y esperanzadores para que pueda regularse una materia tan delicada como es la educación (LGE, 1970, pp. 12526-12527). 
Esta ley propone un cambio en el sistema educativo, ampliando la enseñanza hasta los 14 años con carácter gratuito. La Enseñanza General Básica (EGB) estaba dividida en dos etapas: la primera constituida por cinco cursos, desde 6 a 11 años, y la segunda 3 cursos, desde los 11 a los 14 años. En el artículo 15 se establece que la finalidad de la EGB es "proporcionar una formación integral fundamentalmente igual para todos y adaptada, en lo posible, a las aptitudes y capacidades de cada uno" (LGE, 1970, p.12529). Por ello, integra la Educación Especial en el sistema educativo como respuesta a la demanda por parte de los sectores afectados, que hasta ese momento habían estado bajo la responsabilidad del Patronato Nacional de Educación Especial (Castaño, 2009).

Se advierte especial preocupación por el aprovechamiento académico, afirmando, en el artículo 126, que el alumnado pierde su condición de estudiante por el incumplimiento de la disciplina académica y añade que "reglamentariamente se establecerá el correspondiente cuadro de faltas y sanciones" (LGE, 1970, p. 12540). Señala, además, que corresponde al Consejo de Dirección del centro resolver los problemas de disciplina que afecten al alumnado y que "los educadores tendrán derecho a intervenir en cuanto afecte a la vida, actividad y disciplina de sus respectivos centros docentes a través de los cauces reglamentarios" ( $\mathrm{p}$. 12538).

\subsection{La Ley Orgánica de Estatutos de Centros Escolares de 1980 (LOECE)}

Tras la aprobación de la Constitución de 1978 se promulga la Ley Orgánica de Estatutos de Centros Escolares (1980), que intenta ajustar la organización de los centros docentes y los derechos y deberes del alumnado, a la nueva situación de España. Establece en los centros escolares órganos de gobierno unipersonales y colegiados, introduciendo un modelo democrático y proponiendo una gestión compartida entre el director y la comunidad escolar (García, 2014).

En cuanto a los problemas de conducta del alumnado, la normativa delimita quién y cómo ocuparse de los conflictos en el centro, regulando el vacío legal de las anteriores. Afirma que corresponde al Consejo de Dirección: "resolver los problemas de disciplina que afectan a los estudiantes (...)" (LOECE, 1980, p. 14635) y que "se especificarán en el estatuto o reglamento de régimen interior de cada centro las faltas de disciplina del alumnado y las correlativas sanciones, así como los órganos o sujetos del centro competentes para imponerlas" (p. 14636). Además, solo se podrá expulsar al alumnado cuando su comportamiento suponga un daño grave, asegurando su continuidad educativa, si estuviera cursando un nivel obligatorio (LOECE, 1980).

La ley, que fue derogada en 1982, tomó en consideración las faltas graves del alumnado y las correspondientes sanciones, pero no proponía ninguna actuación ante las faltas leves de los estudiantes, entre las que se encuentran las llamadas conductas disruptivas.

\subsection{Ley Orgánica reguladora del Derecho a la Educación de 1985 (LODE)}

En 1985 se promulgó la Ley Reguladora del Derecho a la Educación (LODE), que organizó la red de centros docentes según su titularidad en públicos, privados y concertados.

En el artículo 42 desarrolla las orientaciones sobre la disciplina del centro escolar y establece que el Consejo Escolar es quien debe "resolver los conflictos e imponer las sanciones al alumnado, de acuerdo con las normas que regulen los derechos y deberes de los 
mismos" (LODE, 1985, p. 21019), y en el artículo 57 añade que este órgano se encargará de resolver los asuntos de carácter grave que se planteen en el centro.

Esta normativa sigue actualmente vigente y ha sido modificada en ocho ocasiones, aunque con poca variación en cuanto al tema tratado. La tabla 2 recoge la modificación de esta ley y los cambios más significativos referentes a los problemas de comportamiento del alumnado en el centro educativo.

Tabla 2. Modificaciones de la LODE en relación a los problemas de comportamiento

\begin{tabular}{ll}
\hline Año & \multicolumn{1}{c}{ Modificaciones sobre los problemas de comportamientos } \\
\hline 1985 & Consejo Escolar encargado de resolver conflictos e imponer las sanciones al \\
1990 & alumnado (art. 42) y resolver asuntos de carácter grave (art. 57) \\
1995 & No hay modificación al respecto \\
1999 & Se elimina el artículo 42 y lo recoge la LOPEG \\
2002 & No hay modificación al respecto \\
2004 & La resolución de los conflictos graves pasa a ser función del director (art. 54) \\
& Modifica y añade aspectos en base a la Ley Orgánica de Medidas de Protección \\
2006 & Integral contra la Violencia de Género (2004) \\
2011 & Añade que es deber básico de los estudiantes, respetar las normas de organización, \\
2013 & convivencia y disciplina del centro (art. 6.4.g. eliminado en 2004) \\
& No hay modificación al respecto \\
& Propone iniciativas que favorezcan la convivencia, la igualdad, no discriminación, \\
& resolución pacífica de conflictos y prevención de violencia de género (art. 57.n) \\
\hline
\end{tabular}

Nota: elaboración propia.

\subsection{Ley Orgánica de Ordenación General del Sistema Educativo de 1990 (LOGSE)}

La Ley Orgánica 1/1990 de 3 de octubre de Ordenación General del Sistema Educativo, más conocida como LOGSE, nace a tenor de los numerosos cambios culturales acaecidos para dar respuesta a cuestiones como "la carencia de configuración educativa en el periodo previo a la escolarización obligatoria o al desfase entre la conclusión de esta y la edad mínima para la incorporación laboral" (LOGSE, 1990, p. 28928). Para ello, amplía la obligatoriedad de la enseñanza hasta los 16 años y cambia la estructura del sistema escolar, organizándola en Infantil, Primaria y Secundaria.

Sustituyó el término de Educación Especial por el de Necesidades Educativas Especiales, "con el fin de hacer realidad los principios de normalización educativa e integración escolar (...) estableciendo el principio de diversidad" (Castaño, 2009, p. 407), según el cual se debían atender las necesidades de cada estudiante en un sistema común para todos.

En cuanto al tema tratado, el artículo 2.3 de esta Ley establece que la actividad educativa se desarrollará atendiendo a diferentes principios entre los que se encuentra: "e) El fomento de los hábitos de comportamiento democrático" (LOGSE, 1990, p. 28930), persiguiendo entre otros fines "b) La formación en el respeto de los derechos y libertades fundamentales y en el ejercicio de la tolerancia y de la libertad dentro de los principios democráticos de convivencia" y "g) La formación para la paz, la cooperación y la solidaridad entre los pueblos" (LOGSE, 1990, p. 28930). Se observa su insistencia en la educación para la convivencia, la paz y el respeto, pero no plantea ninguna sanción ante cualquier conflicto o problema de comportamiento que pueda surgir en el aula. Cinco años más tarde, el Real Decreto 732/1995 de 5 de mayo de 1995 por el que se establecen los derechos y deberes de los alumnos y las normas de convivencia en los centros, especificó que debía ser el Consejo 
Escolar quien se ocupara de la resolución de conflictos a través de una comisión de convivencia. Distinguirá entre conductas contrarias a las normas de convivencia y conductas gravemente perjudiciales para la convivencia del centro, para las que dará pautas concretas a seguir en cuanto a la sanción.

\subsection{Ley Orgánica de la Participación, la Evaluación y el Gobierno de los centros docentes de 1995 (LOPEG)}

Esta Ley que se hace pública el 20 de noviembre de 1995 profundiza lo establecido por la LODE (1985) en lo referente a la participación de la comunidad educativa en la organización y la evaluación del centro, así como en las funciones de los órganos de gobierno de los centros públicos, ajustándolo a lo dispuesto en la LOGSE (1990).

En el artículo 11 indica que el Consejo Escolar del centro, deberá “e) resolver los conflictos e imponer las correcciones con finalidad pedagógica que correspondan a aquellas conductas del alumnado que perjudiquen gravemente la convivencia en el centro, de acuerdo con las normas que establezcan las Administraciones educativas" (LOPEG, 1995, p. 33655). Así, la comunidad educativa como corresponsable de la resolución de conflictos, facilitará la eficacia educativa de las correcciones. Además, en la disposición adicional segunda sobre la escolarización del alumnado con necesidades educativas especiales (NEE), se ofrece atención educativa específica al que presente trastornos graves de conducta, sin mencionar la actuación ante las faltas leves, lo que presupone que es el profesor quien debe resolverlas.

\subsection{Ley Orgánica de Calidad de Educación de 2002 (LOCE)}

La Ley Orgánica 10/2002, de 23 de diciembre, de Calidad de la Educación (LOCE) establece en su exposición de motivos la importancia del valor del esfuerzo personal como condición fundamental para la mejora de la enseñanza y propone un consenso social sobre "ciertas normas y comportamientos (...) que contribuyen al buen funcionamiento de los centros educativos y favorecen su rendimiento" (LOCE, 2002, p. 45189).

Esta ley contempla la responsabilidad del alumnado e insiste en el respeto a las normas de convivencia y la consolidación de los hábitos de disciplina, como condiciones necesarias para el aprendizaje y el desarrollo personal, deberes básicos en las tres etapas educativas (LOCE, 2002).

El director del centro deberá "resolver los conflictos e imponer todas las medidas disciplinarias que correspondan al alumnado, de acuerdo con las normas que establezcan las Administraciones educativas y el cumplimiento de los criterios fijados en el reglamento de régimen interior del centro" (LOCE, 2002, pp. 45207-45208). El Consejo Escolar se encargará de "conocer la resolución de conflictos disciplinarios y la imposición de sanciones y velar por que estas se atengan a la normativa vigente" (LOCE, 2002, p. 45208). El claustro de profesores será informado por el director de la aplicación del régimen disciplinario del centro.

En la sección cuarta se hace especial mención al alumnado con necesidades educativas especiales, incidiendo en el principio de no discriminación y normalización, tenido ya en cuenta por la LOGSE (1990), e incluye atención especializada para el alumnado con trastornos de personalidad y conducta (LOCE, 2002). 
Esta Ley no llegará a aplicarse en su totalidad, siendo derogada en 2006 con la aprobación de la Ley Orgánica de Educación de 2006 (LOE).

\subsection{Ley Orgánica de Educación de 2006 (LOE)}

La Ley Orgánica 2/2006, de 3 de mayo, de Educación (LOE) apremia a que se proporcione una educación de calidad a todos los ciudadanos de ambos sexos, en todos los niveles del sistema educativo (LOE, 2006, p.17159). En cuanto al tema tratado, resalta la importancia de "la educación para la prevención de conflictos y para la resolución pacífica de los mismos, así como la no violencia en todos los ámbitos de la vida personal, familiar y social" (LOE, 2006, p. 17165).

Indica como fin del sistema educativo "la educación en el ejercicio de la tolerancia y de la libertad dentro de los principios democráticos de convivencia” (LOE, 2006, p. 17165). En el capítulo III, plantea como principio de la Educación Secundaria "desarrollar y consolidar hábitos de disciplina, estudio y trabajo individual y en equipo, como condición necesaria para una realización eficaz de las tareas del aprendizaje y como medio de desarrollo personal" (p. 17169).

El título 1, dedicado a la ordenación de las enseñanzas, indica los objetivos de las distintas etapas educativas en cuanto al desarrollo de las capacidades del alumnado y, en relación al comportamiento, especifica los siguientes (ver tabla 3):

Tabla 3. Objetivos de las distintas etapas educativas en cuanto al desarrollo de las capacidades del alumnado relacionadas con el comportamiento en la LOE, 2006

\begin{tabular}{|c|c|}
\hline Etapa & Objetivos \\
\hline Educación Infantil & $\begin{array}{l}\text { Relacionarse con los demás y adquirir progresivamente pautas } \\
\text { elementales de convivencia y relación social. } \\
\text { Ejercitarse en la resolución pacífica de conflictos (p.17167). }\end{array}$ \\
\hline Primaria & $\begin{array}{l}\text { Adquirir habilidades para la prevención y para la resolución pacífica de } \\
\text { conflictos, que les permitan desenvolverse con autonomía en el ámbito } \\
\text { familiar y doméstico, así como en los grupos sociales con los que se } \\
\text { relacionan (p. 17168). }\end{array}$ \\
\hline Secundaria & $\begin{array}{l}\text { Fortalecer sus capacidades afectivas en todos los ámbitos de la } \\
\text { personalidad y en sus relaciones con los demás, así como rechazar la } \\
\text { violencia, los prejuicios de cualquier tipo, los comportamientos sexistas } \\
\text { y resolver pacíficamente los conflictos (p. 17169). }\end{array}$ \\
\hline Bachillerato & $\begin{array}{l}\text { Consolidar una madurez personal y social que les permita actuar de } \\
\text { forma responsable y autónoma y desarrollar su espíritu crítico. Prever y } \\
\text { resolver pacíficamente los conflictos personales, familiares y sociales } \\
\text { (p.17172). }\end{array}$ \\
\hline Formación Profesional & $\begin{array}{l}\text { Aprender por sí mismos y trabajar en equipo, así como formarse en la } \\
\text { prevención de conflictos y en la resolución pacífica de los mismos en } \\
\text { todos los ámbitos de la vida personal, familiar y social (p.17174). }\end{array}$ \\
\hline Formación de adultos & $\begin{array}{l}\text { Prever y resolver pacíficamente los conflictos personales, familiares y } \\
\text { sociales. Fomentar la igualdad efectiva de derechos y oportunidades } \\
\text { entre hombres y mujeres, así como analizar y valorar críticamente las } \\
\text { desigualdades entre ellos (p. 17178) }\end{array}$ \\
\hline
\end{tabular}

Nota: elaboración propia.

El director del Centro educativo, el Consejo Escolar y el Claustro de Profesores serán los órganos encargados de velar por el buen funcionamiento de la normativa vigente sobre resolución de conflictos. En la tabla 4 aparecen sus funciones a este respecto. 
Tabla 4. Funciones de los órganos colegiados en referencia a la resolución de conflictos

\begin{tabular}{ll}
\hline Órgano colegiado & \multicolumn{1}{c}{ Función } \\
\hline \multirow{2}{*}{ Director } & $\begin{array}{l}\text { Favorece la convivencia en el centro, garantizando la mediación en la } \\
\text { resolución de los conflictos e impone las medidas disciplinarias al } \\
\text { alumnado, en cumplimiento de la normativa vigente (p.17191). }\end{array}$ \\
& $\begin{array}{l}\text { Conoce la resolución de conflictos disciplinarios y vela porque se } \\
\text { atengan a la normativa vigente; revisa las decisiones del director } \\
\text { cuando la conducta del alumnado perjudique de forma grave al centro }\end{array}$ \\
& escolar, pudiendo proponer las medidas oportunas (p. 17190). \\
Consejo Escolar & $\begin{array}{l}\text { Propone medidas e iniciativas que favorezcan la convivencia en el } \\
\text { centro, la igualdad entre hombres y mujeres y la resolución pacífica } \\
\text { de conflictos en todos los ámbitos de la vida personal, familiar y } \\
\text { social (p. 17190). }\end{array}$ \\
\hline Claustro de Profesores & Conoce la resolución de conflictos disciplinarios y la imposición de \\
& sanciones y vela porque se atengan a la normativa vigente (p.17191). \\
\hline
\end{tabular}

Nota: elaboración propia.

Se observa que el director posee la máxima autoridad para imponer las medidas disciplinarias ante un conflicto, mientras que el Consejo Escolar será su colaborador para acabar con conductas muy graves. La autoridad del docente en este ámbito queda reducida a controlar que las medidas disciplinarias impuestas se lleven a cabo. Esto entorpece su labor, ya que, para poder abordar cualquier problema de comportamiento en el aula, debe remitirlo a los órganos colegiados antes citados, ralentizando el proceso y haciéndolo ineficaz en la mayoría de los casos, pues muchas de las conductas disruptivas requieren una solución inmediata en el aula.

Según esta ley, todos los centros deben incluir en su proyecto educativo un Plan de Convivencia y establecer normas que garanticen su cumplimiento. Para ello dispone que sean las Administraciones de las Comunidades Autónomas las que establezcan el marco regulador para que los centros elaboren sus proyectos educativos.

Esta ley se modifica en dos ocasiones en 2011, sin cambios significativos.

\subsection{Texto consolidado de la Ley Orgánica para la Mejora de la Calidad Educativa de 2013 (LOMCE) con la LOE (2006)}

En 2013 se aprueba la Ley Orgánica 8/2013, de 9 de diciembre, para la Mejora de la Calidad Educativa (LOMCE), que modifica en un artículo único a la LOE. El texto consolidado de ambas leyes es hoy la ley vigente en España. La última modificación del texto se ha realizado el 23 de marzo de 2018.

Se observa que esta normativa presta especial atención a "la educación para la prevención de conflictos y la resolución pacífica de los mismos (...) en especial en el del acoso escolar" (Legislación consolidada, LOMCE-LOE, 2013, última modificación, p. 11).

En el artículo 124, dedicado a las normas de organización, funcionamiento y convivencia, se mantiene la orden a los centros de elaborar un plan de convivencia que concrete derechos y deberes del alumnado y las medidas correctoras si estos se incumplieran.

Por primera vez, se incide en el carácter educativo, personalizador y recuperador de dichas medidas, garantizando el respeto a todo el alumnado y promoviendo la mejora de las relaciones interpersonales de la comunidad educativa. Diferencia de forma clara entre faltas 
graves y leves, indicando que las medidas correctoras deberán ser proporcionadas a las faltas cometidas. Advierte que la falta será considerada como muy grave, con expulsión temporal o definitiva del centro educativo, cuando las conductas realizadas:

Atenten contra la dignidad personal de otros miembros de la comunidad educativa, que tengan como origen o consecuencia una discriminación o acoso basado en el género, orientación o identidad sexual, o un origen racial, étnico, religioso, de creencias o de discapacidad, o que se realicen contra el alumnado más vulnerable por sus características personales, sociales o educativas (Legislación consolidada LOMCE-LOE, 2013, última modificación, p. 46).

Mientras que "las decisiones de adoptar medidas correctoras por la comisión de faltas leves serán inmediatamente ejecutivas" (Legislación consolidada LOMCE-LOE, 2013, última modificación, p. 46) y las actuaciones educativas se concretarán en los planes de convivencia de los centros educativos "tomando en consideración su situación y condiciones personales" (Legislación consolidada LOMCE-LOE, 2013, última modificación, p. 46) y siguiendo las directrices legislativas, tanto a nivel estatal como las específicas de las comunidades autónomas. En la mayoría de estas se crearon los Observatorios de Convivencia entre los años 2006 y 2007, encargados de:

La coordinación, la consulta, el análisis y la evaluación del estado de la convivencia escolar, para [...] fomentar su mejora, organizar actividades preventivas o de difusión de buenas prácticas y publicar documentos e instrumentos que contribuyan tanto a la comprensión general de todos los fenómenos asociados a la convivencia escolar, como a las aplicaciones y soluciones prácticas en las aulas (Gómez, 2011, pp. 151 y 152).

Medidas más específicas se recogen en los planes de convivencia elaborados en cada centro, que incluirán la programación general anual con las actividades de fomento de la convivencia y las medidas correctoras (Legislación consolidada LOMCE-LOE, 2013, última modificación).

\section{Conclusiones}

Las transformaciones de la sociedad actual han afectado de forma particular a la comunidad educativa, donde el alumnado refleja estos cambios a veces de la forma menos adecuada, se siente inseguro, desconcertado, tiene deseo de saber, pero no tolera el fracaso (De la Fuente, 2012). Todo ello ha incrementado la indisciplina en el aula, provocando que el profesorado dedique mucho tiempo a resolver estos problemas de comportamiento (OCDE, 2014).

Para poder hacer frente a esta problemática resulta imprescindible distinguir los tipos de conflictos que se generan en la escuela, desde los más graves, provocados por conductas violentas y agresivas contra el profesorado y alumnado, hasta las conductas disruptivas, menos graves, como levantarse, gritar, cantar o desobedecer las órdenes del docente, que impiden el buen desarrollo del proceso enseñanza-aprendizaje. Esto facilitará la utilización de las medidas educativas adecuadas a cada tipo de conflicto (Gotzens et al., 2015).

A pesar de ello, en la revisión de la legislación educativa realizada, se ha podido observar la evolución en la atención creciente ante los problemas graves de comportamiento, pero no así ante aquellas conductas que ocasionan malestar y son una preocupación constante 
para el profesorado. La LOMCE (2013) aborda estos comportamientos disruptivos, señalados como faltas leves, aunque sin dar pautas concretas de actuación.

Al analizar la normativa educativa se ha observado que la Ley de Instrucción Pública (1857) no afronta el problema de la disciplina, pues en ese momento eran pocos y muy motivados los que accedían a la educación, y su preocupación era disminuir la tasa de analfabetismo del país. Sin embargo, la Ley General de Educación (1970) legisla que la mala conducta es motivo suficiente para que el alumnado pierda su condición de estudiante y corresponde al Consejo de Dirección del centro resolver cualquier problema de disciplina.

Con la promulgación de la LODE (1985), esta función pasará a formar parte del Consejo Escolar, que, además, se ocupará de imponer sanciones ante los conflictos graves. Posteriormente, en la quinta actualización de esta ley, en 2004, se añaden medidas de protección integral contra la violencia de género.

La LOE (2006) establece entre los objetivos de las distintas etapas educativas la adquisición de habilidades para la prevención y resolución de conflictos. Este fin se concreta aún más en 2013, en el texto consolidado LOE-LOMCE, vigente actualmente, en la que se incide en lo referido al acoso escolar y las conductas que atentan contra la dignidad personal de otros miembros de la comunidad educativa, considerándolas como graves. Se tomarán medidas correctoras importantes como la expulsión temporal o definitiva del centro para las faltas graves.

Se advierte, en definitiva, una evolución histórica de las leyes educativas en su preocupación ante los conflictos, mostrando así la toma de conciencia por parte de la sociedad del grave perjuicio que supone el aumento de esta situación en la escuela.

Actualmente, en la ley vigente, se hace referencia y se concretan las medidas de resolución de los conflictos graves, aquellos que tienen que ver con conductas violentas, mientras que las medidas para resolver las conductas disruptivas, contenidas en las llamadas faltas leves, quedan en manos de los centros educativos, que deberán reflejarlo en los planes de convivencia. Estas son cada vez más frecuentes y provocan numerosos problemas a los docentes. Además, como indican Gotzens et al. (2015), en muchos casos el profesorado cuenta con escasa experiencia de formación en el tema y las estrategias utilizadas como castigo son a menudo intuitivas, basadas en lo que se aplicaba en tiempos pasados y poco eficientes para el alumnado actual, que no las percibe como corrección y, por tanto, persiste en su comportamiento disruptivo.

Por ello, aunque a nivel legislativo parece estar cada vez más clara la diferencia entre las conductas disruptivas y aquellas de problemática más grave como bullying o violencia escolar, para las que sí hay un protocolo claro de actuación, sería necesario establecer medidas claras para abordar las conductas disruptivas que, aun siendo menos graves, merecen una solución, pues no se puede obviar que suponen un obstáculo para el buen funcionamiento de los centros y del proceso enseñanza-aprendizaje. El tratamiento específico de este tipo de conductas mejoraría además las relaciones interpersonales y el clima general del aula, provocando una mayor motivación del alumnado hacia el aprendizaje y del profesorado hacia su trabajo (Gotzens, Badia, Genovard y Dezcallar, 2015; Gómez y Cuña, 2017). 


\section{Referencias bibliográficas}

Badia, M., Cladellas, R., Gotzens, C. y Muntada, M. (2012). La indisciplina social e instruccional en adolescentes y universitarios: la influencia del género. Electronic Journal of Research in Educational Psychology, 10 (2), 693-712. Recuperado de http://www.redalyc.org/articulo.oa?id=293123547009

Castaño, A. M. (2009). La atención a la diversidad en el marco de una escuela inclusiva: evolución histórica. En M.R. Berruezo y S. Conejero (Coords.), El largo camino hacia una educación inclusiva: la educación especial y social del siglo XIX a nuestros días: XV Coloquio de Historia de la Educación (pp. 405-416). Pamplona-Iruñea, España: Universidad Pública de Navarra.

De la Fuente, D. (2012). Los cambios sociales y su reflejo en la educación. Propuestas educativas desde la asignatura de música. Dedica. Revista de Educação e Humanidades, 2, 249-260. Recuperado de https://bit.ly/2sFIYPz

De Puelles, M. (2011). La educación secundaria en la España democrática. Antecedentes, problemas y perspectivas. Cuadernos de Pesquisa, 41 (144), 710-731. Recuperado de https://bit.ly/35yzBzM

Egido, I. (2002). Las instituciones de educación primaria y secundaria. En M. Carreño (Ed.), Teorías e instituciones contemporáneas de educación (pp. 217-250). Madrid: Síntesis.

Esteban, R., Fernández, J.G., Díaz, A. y Contreras, O.R. (2012). Las conductas que alteran la convivencia en las clases de educación física. Revista Internacional de Medicina y Ciencias de la Actividad Física y el Deporte, 12 (47), 459-472. Recuperado de https://bit.ly/2M65ZCj

García, J. (2017). Evolución legislativa de la educación inclusiva en España. Revista Nacional $e$ Internacional de Educación Inclusiva, 10 (1), 251-264. Recuperado de https://bit.ly/2sGTUMT

García Garnica, M. (2014). Evolución de la función directiva en España: recorrido por más de un siglo de historia. Aula de encuentro, 16 (1), 139-155. Recuperado de https://bit.ly/2M75Caz

Gómez, F. A. (2011). La normativa sobre convivencia escolar en España. Revista AMAzônica, 6(1), 149-185. Recuperado de https://bit.ly/2rNbXkN

Gómez, M. C. y Cuña, A. R. (2017). Estrategias de intervención en conductas disruptivas. Educação Por Escrito, 8(2), 278-293. doi:10.15448/2179-8435.2017.2

Gotzens, C., Badia, M., Genovard, C. y Dezcallar, T. (2010). Estudio comparativo de la gravedad atribuida a las conductas disruptivas en el aula. Electronic Journal of Research in Educational Psychology, 8 (1), 33-58. Recuperado de https://bit.ly/2r1fBH8

Gotzens, C., Cladellas, R., Clariana, M. y Badía M. (2015). Indisciplina Instruccional y Convencional: su predicción en el Rendimiento Académico. Revista Colombiana de Psicología, 24 (2), 317-330. doi:10.15446/rcp.v24n2.44148 
Ley de Instrucción Pública, de 9 de septiembre, Gaceta de Madrid, $\mathrm{n}^{\circ}$ 1660, 1857, 9 septiembre.

Ley General de Educación y Financiamiento de la Reforma Educativa (LGE) (Ley General 14/1970, 4 de agosto). Boletín Oficial del Estado, nº 187, 1970, 6 agosto.

Ley Orgánica por la que se regula el Estatuto de Centros Escolares (LOECE) (Ley Orgánica 5/1980, 19 de junio). Boletín Oficial del Estado, nº 154, 1980, 27 junio.

Ley Orgánica reguladora del Derecho a la Educación (LODE) (Ley Orgánica 8/1985, 3 de julio). Boletín Oficial del Estado, nº 159, 1985, 4 julio.

Ley Orgánica de Ordenación General del Sistema Educativo (LOGSE) (Ley Orgánica 1/1990, 3 de octubre). Boletín Oficial del Estado, n 238, 1990, 4 octubre.

Ley Orgánica de la participación, la evaluación y el gobierno de los centros docentes (LOPEG) (Ley Orgánica 9/1995, 20 de noviembre). Boletín Oficial del Estado, nº 278, 1995, 21 noviembre.

Ley Orgánica de Calidad de la Educación (LOCE) (Ley Orgánica 10/2002, 23 diciembre). Boletín Oficial del Estado, $n^{\circ}$ 307, 2002, 24 diciembre.

Ley Orgánica Medidas de Protección Integral contra la Violencia de Género (Ley orgánica 1/2004, 28 de diciembre). Boletín Oficial del Estado, nº 313, 2004, 29 diciembre.

Ley Orgánica de Educación (LOE) (Ley Orgánica 2/2006, 3 de mayo). Boletín Oficial del Estado, no 106, 2006, 4 mayo.

Ley Orgánica para la mejora de la calidad educativa (LOMCE) (Ley Orgánica 8/2013, 9 de diciembre). Boletín Oficial del Estado, nº 295, 2013, 10 diciembre.

Ley Orgánica para la mejora de la calidad educativa (LOMCE) (Ley Orgánica 8/2013, 9 de diciembre) Texto consolidado. Última modificación: 23 de marzo de 2018, BOE nº295 (2013)

Ministerio de Educación Cultura y Deporte (2004). Evolución del sistema educativo español. Recuperado de https://bit.ly/2r2Egv3

Montero, A. (2009). La Ley de Instrucción Pública (Ley Moyano, 1857). Cabás: Revista del Centro de Recurso, Interpretación y Estudios en materia educativa (CRIEME), (1). Recuperado de https://bit.ly/2M4Wrr3

OCDE (2014). TALIS 2013 Results: An International Perspective on Teaching and Learning. https://doi.org/10.1787/9789264196261-en

Real Decreto por el que se establecen los derechos y deberes de los alumnos y las normas de convivencia en los centros. (Real Decreto 732/1995, 5 de mayo de 1995). Boletín Oficial del Estado, nº 131, 1995, 2 junio.

Rodríguez, C. (2017). La jurisdicción de menores ante los casos de bullying y ciberbullying. Revista de Estudios de Juventud, 115, 31-54. Recuperado de https://bit.ly/35y37pB 
Valderrama, R. (2017). Modelo de gestión estratégico en la convivencia y disciplina escolar para mejorar el comportamiento en los estudiantes de las instituciones educativas de José Leonardo Ortiz, Chiclayo 2015. Hacedor-Aiapaec. 1 (1), 1-39. Recuperado de https://bit.ly/34t6o83

Villalba, J. (2016). La convivencia escolar en positivo. Educación y Humanismo, 18 (30), 92106. doi:10.17081/eduhum.18.30.1324 
